\title{
Oral Miltefosine Treatment in Children With Visceral Leishmaniasis: a Brief Review
}

\author{
Emilio Palumbo \\ Department of Pediatric, Hospital of Sondrio; Italy
}

\begin{abstract}
Visceral leishmaniasis (VL) or kala-azar is an infection disease caused by hemiflagellate protozoan parasites (Leishmania donovani) and transmitted to humans by the phlebotomine sandfly. Leishmaniasis is distributed worldwide and 13 million people are estimated to be infected, with about 1.8 million new cases each year. All antileishmanial drugs are toxic and most have to be used parenterally for prolonged period. The therapy has been further complicated by large number of infected children and declining effectiveness of pentavalent antimonial compounds. Although the lipid formulations of amphotericin B are an important advance in therapy, their high cost precludes their use. Miltefosine, a phosphocholine analogue originally developed as antimalignant drug, has been found to be highly active against Leishmania in vitro and in animal model. Based on these experiences this drug was tried against human visceral leishmaniasis and found to be highly effective in children. The aim of this review is to evidence the pharmacodymamic and pharmacokinetic characteristics and the safety, tolerance and efficacy of this drug for treatment of visceral leishmaniasis in children.

Key-Words: Miltefosine, treatment, children, Leishmaniasis.
\end{abstract}

Visceral leishmaniasis (VL) or kala-azar is an infection disease caused by hemiflagellate protozoan parasites (Leishmania donovani) and transmitted to human by the phlebotomine sandfly. Leishmaniasis is distributed worldwide and 13 million people are estimated to be infected, with about 1.8 million new cases each year. Approximately $50 \%$ of these patients are children. Furthermore, leishmania has emerged as an opportunistic pathogen of HIV-infected children.

In recent years, the treatment of VL is far from satisfactory. All antileishmanial drugs are toxic and most have to be used parenterally for prolonged period. The therapy has been further complicated by large number of infected children and declining effectiveness of pentavalent antimonial compounds. Although the lipid formulations of amphotericin B are an important advance in therapy, their high cost precludes their use. Miltefosine, a phosphocholine analogue originally developed as antimalignant drug, has been found to be highly active against Leishmania in vitro and in animal model [1,2].

Based on these experiences this drug was tried against human visceral leishmaniasis and found to be highly effective in children. The aim of this review is to evidence the pharmacodymamic and pharmacokinetic characteristics and the safety, tolerance and efficacy of this drug for treatment of visceral leishmaniasis in children.

\section{Mechanism of Action and Pharmacokinetics}

Miltefosine primarily interferes with cellular membranes without interacting with DNA and modulates membrane permeability and fluidity, membrane lipid composition, metabolism of phospholipids and proliferation signal transduction. Miltefosine is well absorbed after oral

Received on 28 October 2007; revised 27 January 2008.

Address for correspondence: Dr. Emilio Palumbo. Via Dell'Arcangelo Michele. 71100 Fogia. Italy. Phone: + 390881 685023. E-mail: emipalu2003@yahoo.it.

The Brazilian Journal of Infectious Diseases 2008;12(1):2-4. (C) 2008 by The Brazilian Journal of Infectious Diseases and Contexto Publishing. All rights reserved. administration and is widely distributed. It has a long half-life of about eight days [3]. Howe ver, little pharmacokinetic data is available in human beings. In rats, miltefosine is rapidly taken up and accumulates in several internal organs, such as kidney, liver, lung, spleen and adrenal glands. On oral administration of miltefosine $30 \mathrm{mg} / \mathrm{Kg}$ of body weight twice per day, concentrations of 155 to $189 \mathrm{nmol} / \mathrm{g}$ of tissue are achieved [4]. Miltefosine is degraded slowly in vivo, with half-life of 96 hours in mice [5]. It is slowly metabolized by phospholipase to form products such as choline and long chain alcohols that are physiological metabolites and can be recycled into phospholipids [6,7]. The dose of miltefosine is $2.5 \mathrm{mg} / \mathrm{Kg} /$ day, preferably in two divided doses or single dose orally for 28 days. The drug is available as $50 \mathrm{mg}$ and $10 \mathrm{mg}$ capsules. Leishmania have high levels of ether-lipids and these are mainly found in the glycosylphosphophatidylinositol anchored glycoprotein and glycolipids present on the surface of the parasites. Miltefosine acts on key enzymes involved in the metabolism of ether lipids. These enzymes include diethylacetonephosphate acetyltransferase, sn-1-acyl-2-lyso-glycero-phosphocholine and sn-1-alkyl-2-lyso-glycero-3-phosphocholine acetyltransferase. The initial steps in the ether-lipid metabolism occur in glycosomes. Miltefosine does not act on the enzymes involved in the initial steps in ether lipid metabolism in Leishmania. However, the metabolism of the latter phosphatidylcholine base intermediates, which are involved in the synthesis of acyl- and alkylglycerophospholipids, are associated with glycosomes. Miltefosine inhibits this glycosomal alkyl specific alkylspecific-acyl CoA acetyltransferase in a dose dependent manner [8].

In vitro studies, it is demonstrated that miltefosine stimulates $\mathrm{T}$ cells and macrophages to secrete activating cytokines, including interferon gamma (IFN $\gamma$ )and enhances macrophage production of microcidal reactive nitrogen and oxygen intermediates. Genetically deficient mice were infected with Leishmania donovani to determine these effects in vivo. 
Intracellular killing was retained in $\mathrm{T}$ cell deficient mice suggesting that miltefosine induced visceral leishmanicidal effect, which does not require host $\mathrm{T}$ cell-dependent or activated macrophage-mediated mechanisms [9].

\section{Efficacy and Tolerability}

Singh U.K. et al. [10] have compared safety, tolerance and efficacy of miltefosine with available gold standard antileishmanial drug, amphotericin B, a parenteral formulation in children with VL. Patients were randomized into four groups. In groups 1 and 2, patients were given miltefosine in a dose of $2.5 \mathrm{mg} / \mathrm{Kg} /$ day orally to a maximum of $100 \mathrm{mg}$ and groups 3 and 4 received amphotericin $B$ at a dose of $1 \mathrm{mg} / \mathrm{Kg} /$ day (total: $15 \mathrm{mg} / \mathrm{Kg}$ ). All patients were followed up at completion of therapy, at three and at six months after the end of the treatment for clinical response, esplenic size and parasitologically. Out of 125 children, 44 were in group 1, 20 in group 2, 38 in group 3 and 23 in group 4; 124 patients had parasitological cure with relapse in one patient of group 1 during follow up. Final cure rate with miltefosine and amphotericin B was 93.2\%, 95\%, $92.1 \%$ and $91.3 \%$ in groups $1,2,3$ and 4 respectively, which are statistically insignificant. Gastrointestinal side effects, as diarrhea and vomiting, were observed in 26 and 23 patients from group 1 and 2, respectively. Miltefosine is safe, well tolerated, and highly effective and has same efficacy as amphotericin B in newly diagnosed and SAG resistant children with visceral leishmaniasis [10].

In an other randomized, open-label study (Sundar S et al., 2006) 29912 years-old patients or older received orally administered miltefosine [ 50 or $100 \mathrm{mg}$ (approximately $2.5 \mathrm{mg}$ per kilogram of body weight) daily for 28 days] and 99 patients received intravenously administered amphotericin B (1 mg per kilogram of body weight every other day for a total of 15 injections). In the end of treatment, splenic aspirates were obtained from 293 patients in the miltefosine group and 98 patients in the amphotericin B group. No parasites were identified, for an initial cure rate of $100 \%$. At six months after the completion of treatment, 282 of the 299 patients in the miltefosine group [94\% (CI 95\%: 91-97)]) and 96 of the 99 patients in the amphotericin B group (97:\%) had not had a relapse; these patients were classified as cured. Vomiting and diarrhea, generally lasting one to two days, occurred in $38 \%$ and in $20 \%$ of the patients in the miltefosine group, respectively. This study evidenced as miltefosine is an effective and safe treatment for visceral leishmaniasis and it may be particularly advantageous because it can be administered orally. It may also be helpful in regions where parasites are resistant to current agents [11].

Sundar et al. studied 39 children (defined as $<12$ years of age) with visceral leishmaniasis, demonstrated by parasites in splenic aspirates, treating them with oral miltefosine daily for 28 days: 21 patients received $1.5 \mathrm{mg} / \mathrm{Kg} /$ day (group A); and 18 patients received $2.5 \mathrm{mg} / \mathrm{Kg} /$ day (group B). All patients were parasitologically negative and symptomatically improved by the end of therapy at day 28 of therapy; the initial parasitologic cure rate was $100 \%$. Two patients in each treatment group relapsed with fever, splenomegaly and parasite-positive splenic aspirates by the end of the 6-month follow-up. The protocol final clinical cure rate was 19 of $21=$ $90 \%$ in group A and 15 of $17=88 \%$ in group B. Miltefosine was well-tolerated. As the adult experience, gastrointestinal adverse events were seen: 33 and $39 \%$ of children experienced vomiting and 5 and $17 \%$ experienced diarrhea in groups $\mathrm{A}$ and $\mathrm{B}$, respectively, but all episodes were mild to moderate in severity and commonly lasted less than one day without symptomatic treatment. In this study, oral miltefosine was safe and had approximately $90 \%$ of effectiveness in this initial clinical trial of childhood visceral leishmaniasis [12].

Bhattacharya et al. evaluated the use of the adult dosage of miltefosine $(2.5 \mathrm{mg} / \mathrm{Kg}$ per day for 28 days $)$ in 80 Indian children (age, 2-11 years) with parasitologically confirmed infection in an open-label clinical trial. Clinical and parasitological parameters were reassessed at the end of treatment and 6 months later. One patient died of intercurrent pneumonia at day 6 . The other 79 patients demonstrated no parasites after treatment, had marked clinical improvement, and were deemed initially cured. Three patients had relapsed, and one patient was lost not found to follow-up. The final cure rate was $94 \%$ for all enrolled patients and $95 \%$ for evaluable patients. Side effects included mild-to-moderate vomiting or diarrhea (each in approximately $25 \%$ of patients) and mild-to-moderate transient elevations in the aspartate aminotransferase level during the early treatment phase (in $55 \%$ ). This trial indicates that miltefosine is as effective and well tolerated in Indian children with VL as in adults and that it can be recommended as the first choice for treatment of childhood VL [13].

\section{Conclusion}

Miltefosine, a phosphocholine analogue originally developed as antimalignant drug, has been found to be highly active against Leishmania in vitro and animal model. Based on these experiences, this drug was tried against human visceral leishmaniasis and it was found to be highly effective in children. Studies in literature evidenced as miltefosine is an effective and safe treatment for visceral leishmaniasis (approximately 90\% effective in childhood visceral leishmaniasis) and it may be particularly advantageous because it can be administered orally. It may also be helpful in regions where parasites are resistant to current agents.

\section{References}

1. Croft S.L., Neal R.A., Pendergast W., Chan J.H. The activity of alkylphosphocholines and related deravatives against Leishmania donovani. Biochemical Parasitol 1987;2633-6.

2. Kuhlencord A., Maniera T., Eibl H., Unger C. Hexadecyl Phosphocholine; oral treatment of visceral leishmaniasis in mice. Antimicrobial agents and Chemotherapy 1992;36:1630-4.

3. Sunder S., Jha T.K., Thakur C.P., et al. Oral Miltefosine for Indian Visceral leishmaniasis. $N$ Engl J Med 2002;347:1739-46. 
4. Jha T.K., Sundar S., Thakur C.P., et al. Miltefosine, an oral agent, for the treatment of Indian visceral leishmaniasis. N Engl J Med 1999;341:1795-800.

5. Bresiser A., Kim D.J., Fleer E.A., et al. Distribution and metabolism of hexadecylphosphocholine in mice. Lipids 1987;22:925-6.

6. Seifert K., Duchaene M., Werndorfer W.H. Effects of miltefosine and other alkylphosphocholines on human ingtestinal parasite Entamoeba histolytica. Antimicrob Agents Chemother 2001; 45 : $1505-10$.

7. Mohan A., Seth S. Oral miltefosine in treatment of kala azar. Nat Med J Ind 2000;13:202-3.

8. Lux H., Heise N., Klenner T., et al. Ether - lipid (alkylphospholipid) matabolism and the mechanism of action of ether - lipid analogues in visceral leishmania. Mol Biochem Parasitol 2000;111:1-14
9. Murray H.W., Delph- Etienne S. Visceral leishmanicidal activity of hexadecylphosphocholine (miltefosine) in mice deficient in Tcells and activated macrophage microcidal mechanisms. J Inf Dis 2000; $181: 795-9$.

10. Singh U.K., Prasad R., Mishra O.P., Jayswal B.P. Miltefosine in children with visceral leismaniasisi: a prospective, multicentric, cross-sectional study. Indian J Pediatr 2006;73(12):1077-80.

11. Sundar S., Jha T.K., Thakur C.P., et al. Oral miltefosine for the treatment of Indian visceral leishmaniasis. Trans R Soc Trop Med Hyg 2006; 100 suppl 1:S26-33.

12. Sundar S., Jha T.K., Sindermann H., et al. Oral miltefosine treatment in children with mild to moderate Indian visceral leishmaniasis. Pediatr J Infect Dis 2003;22(5):434-8.

13. Bhattachrya S.K., Jha T.K., Sundar S., et al. Efficacy and tolerability of miltefosine for childhood visceral leishmaniasisi in India. Clin Infect Dis 2004;38(2):217-21 